\section{OPEN ACCESS}

Edited by:

Anna Karolina Kiss,

Medical University of Warsaw, Poland

Reviewed by:

Michał Tomczyk,

Medical University of Białystok

Poland

Ouyang Chen,

Second Military Medical University,

China

${ }^{*}$ Correspondence:

Jun Sheng

shengj@ynau.edu.cn

Xuanjun Wang

wangxuanjun@gmail.com

Huanhuan Xu

xuhuan1017@163.com

Specialty section:

This article was submitted to

Ethnopharmacology,

a section of the journal

Frontiers in Pharmacology

Received: 18 March 2017 Accepted: 15 May 2017

Published: 31 May 2017

Citation:

Liu T, Ding S, Yin D, Cuan X, Xie C, $X u H$, Wang $X$ and Sheng J (2017)

Pu-erh Tea Extract Ameliorates Ovariectomy-Induced Osteoporosis in Rats and Suppresses

Osteoclastogenesis In Vitro. Front. Pharmacol. 8:324. doi: 10.3389/fphar.2017.00324

\title{
Pu-erh Tea Extract Ameliorates Ovariectomy-Induced Osteoporosis in Rats and Suppresses Osteoclastogenesis In Vitro
}

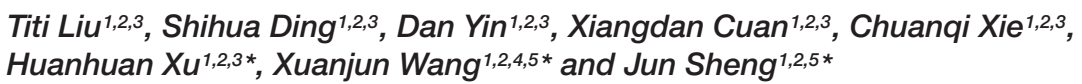

${ }^{1}$ Key Laboratory of Pu-erh Tea Science, Ministry of Education, Yunnan Agricultural University, Kunming, China, ${ }^{2}$ Tea Research Center of Yunnan, Kunming, China, ${ }^{3}$ College of Food Science and Technology, Yunnan Agricultural University, Kunming, China, ${ }^{4}$ College of Longrun Pu-erh Tea, Yunnan Agricultural University, Kunming, China, ${ }^{5}$ State Key Laboratory for Conservation and Utilization of Bio-Resources in Yunnan, Kunming, China

Background and Objective: Tea drinking is associated with positive effects on bone health and may protect against osteoporosis, especially in elderly women. Pu-erh tea has many beneficial effects on human health; however, whether Pu-erh tea has antiosteoporotic potential remains unclear. Thus, we investigated the effects of Pu-erh tea extract (PTE) on ovariectomy-induced osteoporosis in rats and on osteoclastogenesis in vitro.

Methods: Female Wistar rats were divided into six groups: the sham, model, and Xian-Ling-Gu-Bao capsule (XLGB) groups, and the low-, medium-, and high-dose PTE groups. Ovariectomized (OVX) rats were used as an animal model of osteoporosis. The animals were intragastrically administered distilled water, XLGB, or different concentrations of PTE for 13 weeks. Body weight, blood biochemical indicators, relative organ coefficients, femoral bone mineral density (BMD), bone biomechanical properties, and bone microarchitecture were examined and analyzed. Additionally, the in vitro effects of PTE on osteoclastic activities were investigated using the RAW 264.7 cell line as an osteoclast differentiation model. The effects of PTE on osteoclast differentiation and the expression of osteoclast-specific genes and proteins were determined.

Results: PTE reduced OVX-induced body weight gain after 6 weeks of treatment, and the high-dose exerted a significant effect. High-dose PTE significantly ameliorated OVXinduced estradiol $\left(E_{2}\right)$ deficiency. PTE treatment maintained calcium and phosphorus homeostasis and improved other blood biochemical parameters to various degrees. In addition, PTE treatment improved organ coefficients of the femur, uterus, and vagina and improved femoral BMD and bone biomechanical properties. PTE treatment strikingly ameliorated bone microarchitecture. Moreover, in the in vitro studies, osteoclast differentiation using the differentiation cell model was significantly inhibited by PTE without cytotoxic effects. Additionally, PTE efficaciously suppressed the expression of key osteoclast-specific genes and proteins.

Conclusion: PTE can ameliorate ovariectomy-induced osteoporosis in rats and suppress osteoclastogenesis in vitro.

Keywords: PTE, osteoporosis, ovariectomy, bone quality, osteoclastogenesis 


\section{INTRODUCTION}

Osteoporosis, a systemic bone metabolic disease, is caused by many factors and is characterized by decreased bone mass, bone density, and microarchitectural deterioration of bone tissue, which results in enhanced bone fragility and an increased susceptibility to fractures (Park et al., 2012; Byun et al., 2014; Roux and Briot, 2017). It has been ignored by most people because patients with osteoporosis do not have specific symptoms in the clinic, and pathogenesis is very slow (Das et al., 2004; Li et al., 2017). Currently, osteoporosis has become a serious threat to human health and eventually leads to reduced daily activity, lowered quality of life, and increased mortality (Shen et al., 2013; Tabatabaei-Malazy et al., 2017). With an increase in the aging population and life expectancy, osteoporosis has become more prevalent in older individuals worldwide (Shen et al., 2013). In particular, women are prone to develop osteoporosis after menopause because of accelerated bone turnover that is secondary to estrogen deficiency, resulting in an increased risk of fractures (Tabatabaei-Malazy et al., 2017). However, the pathogenesis of osteoporosis has not been fully elucidated. Moreover, drug treatment for osteoporosis has some limitations and may cause adverse effects in patients (Schwarz et al., 2014; Lee et al., 2016). Therefore, effective preventative measures, which involve many long-term strategies, including healthy dietary supplementation and regular physical activity, are imperative for individuals at risk of developing osteoporosis (Kleerekoper, 2006; Park et al., 2012; Michael Lewiecki, 2017).

Tea is one of the most widely consumed beverages all over the world, second only to water. Emerging studies show that tea drinking has promising protective effects on human health, and its pharmacological effects and safety have been confirmed (Xu et al., 2016). Various types of tea can be produced depending on the processing method, including green tea, black tea, and $\mathrm{Pu}$-erh tea. Notably, $\mathrm{Pu}$-erh tea is a distinctive Chinese tea made from crude green tea leaves by postfermentation with microorganisms. It is mainly produced in the Yunnan Province of China. Water extracts from $\mathrm{Pu}$-erh tea contain abundant bioactive constituents and have shown various biological activities, including antiobesity, hypolipidemic, antidiabetic, anti-inflammatory, and anticancer properties (Yu et al., 2014; Cai et al., 2016; Wang et al., 2016).

Convincing evidence from in vitro, animal, and human studies using various bone loss models strongly suggests that tea drinking is closely associated with bone health and may protect against osteoporosis and osteoporotic fracture, especially in middle-aged and elderly individuals (Hegarty et al., 2000; Devine et al., 2007; Shen et al., 2013; Ng et al., 2014; Huang and Tang, 2016; Nash and Ward, 2017). Living bone is a rigid yet dynamic organ that is continuously molded, shaped, and repaired (Boyle et al., 2003). Bone homeostasis is maintained through a balance between bone formation and bone resorption. During bone remodeling, excessive osteoclastic activity and inadequate osteoblastic activity significantly contribute to osteoporosis development (Shen et al., 2013; Zeng et al., 2016). Green tea extract and bioactive components found in it, especially tea polyphenols, could effectively improve bone quality by stimulating osteoblast differentiation and suppressing osteoclast formation and differentiation (Shen et al., 2009, 2011; Oka et al., 2012; Byun et al., 2014; Vester et al., 2014). Furthermore, black tea water extract was shown to increase serum estradiol $\left(\mathrm{E}_{2}\right)$ levels and prevent against estrogen deficiency-related osteoporotic damage in an oophorectomized rat model of osteoporosis (Das et al., 2005). However, whether $\mathrm{Pu}$-erh tea has anti-osteoporotic potential has not been well elucidated.

To investigate whether Pu-erh tea extract (PTE) could be used to prevent osteoporosis, the present study was designed to evaluate the pharmacological effect of PTE in preventing osteoporosis using ovariectomized (OVX) female rats as an animal model of postmenopausal osteoporosis. Furthermore, we investigated the in vitro effects of PTE on osteoclastic activities using a standard osteoclast differentiation model, which will aid in understanding the molecular mechanisms underlying the protective properties of PTE against osteoporosis.

\section{MATERIALS AND METHODS}

\section{Reagents and Antibodies}

Fermented Pu-erh tea was kindly provided by the China Academy of Pu-erh Tea Research (Yu et al., 2014; Cai et al., 2016). E. coli-derived recombinant mouse RANKL was obtained from R\&D Systems (Minneapolis, MN, United States) and dissolved in $0.1 \%$ bovine serum albumin (BSA) in phosphatebuffered saline (PBS). Ten percent neutral formaldehyde was obtained from Jinan Biological Technology Co., Ltd, China. Xian-Ling-Gu-Bao (XLGB) capsules were purchased from Guizhou Tongjitang Pharmaceutical Co., Ltd, China. Alkaline phosphatase (ALP), calcium, and phosphorus assay kits were purchased from Zhong-Sheng BeiKong Bio-technology and Science Inc., China. Bone gla protein (BGP) and $\mathrm{E}_{2}$ radioimmunoassay kits were purchased from the Beijing North Institute of Biological Technology (BNIBT), China. Rat interleukin (IL)-1 $\beta$ and IL-6 ELISA kits were purchased from Neobioscience Biological Technology Co., Ltd, China. 3-(4,5Dimethylthiazol-2-yl)-2,5-diphenyltetrazolium bromide (MTT) was purchased from Sigma-Aldrich (St. Louis, MO, United States). The acid phosphatase (ACP) assay kit and tartrateresistant acid phosphatase (TRAP) staining kit were obtained from Nanjing Jiancheng Bioengineering Institute (Nanjing, China). Anti-NFATc1, anti-c-Src, anti-cathepsin K, and anti-cFos antibodies were purchased from Santa Cruz Biotechnology (Santa Cruz, CA, United States). Anti- $\beta$-tubulin and horseradish peroxidase-conjugated secondary antibodies were purchased from Proteintech Group, Inc. (Rosemont, IL, United States) and Thermo Fisher Scientific (Waltham, MA, United States), respectively.

\section{Preparation of Pu-erh Tea Extract}

$\mathrm{Pu}$-erh tea extract was prepared as previously described (Zhao H. et al., 2011; Zhao L. et al., 2011; Xie et al., 2017). Briefly, the required fermented $\mathrm{Pu}$-erh tea amounts were boiled in 
water for $30 \mathrm{~min}$ three times. The decoction was then collected, concentrated and spray-dried. The PTE powder was dissolved in distilled water, and the $\mathrm{pH}$ was adjusted to 7.4 with $1 \mathrm{M}$ $\mathrm{NaOH}$. Analysis of the chemical composition of PTE found that caffeine, tea saccharide, and especially polyphenol were the major components, accounted for 4.18, 9.31, and 33.13\%, respectively. Tea pigments [also regarded as oxidative tea polyphenols (OTP)], including theaflavins, thearubigins, and theabrownins, were abundant in the fermented Pu-erh tea (Zhao L. et al., 2011; Xie et al., 2017). Moreover, OTP were the major bioactive constituents in the PTE (Huang et al., 2014; Shan et al., 2016; Wang et al., 2016; Lu et al., 2017).

\section{Animals}

Healthy specific-pathogen-free (SPF) female Wistar rats [12 weeks old, Cat No. SCXK-(Ji)2007-0003] from the Laboratory Animal Center of Jilin University were used in the animal experiments. All rats were housed in polypropylene cages with sterile paddy husk and were maintained under a controlled environment (humidity 50-60\%, ambient temperature $24 \pm 1^{\circ} \mathrm{C}$, $12 \mathrm{~h}$ light/dark cycle). During this study, OVX rats were pair-fed a normal diet based on the average weekly food consumption of the sham control group. All experimental procedures were performed in accordance with the guidelines of the Yunnan Agricultural University Committee for Care and Use of Laboratory Animals and were approved by the Animal Experiments Ethics Committee of Yunnan Agricultural University.

\section{Group Designations and Treatment Administration}

After 1 week of feeding adaptation, all rats, which were anesthetized with chloral hydrate, were either sham-operated (sham, $n=12$ ) or ovariectomized (OVX, $n=70$ ). The OVX rats were randomly divided into the model group (model, $n=14$ ), XLGB capsule group (XLGB, $n=14 ; 240 \mathrm{mg} / \mathrm{kg}$ body weight/day), low-dose PTE group (low-dose, $n=14 ; 120 \mathrm{mg} / \mathrm{kg}$ body weight/day), medium-dose PTE group (medium-dose, $n=14 ; 370 \mathrm{mg} / \mathrm{kg}$ body weight/day), and high-dose PTE group (high-dose, $n=14 ; 500 \mathrm{mg} / \mathrm{kg}$ body weight/day). The animals were monitored for 15 days before initiating the therapeutic regimen to allow them to recover from the operation. The animals were continuously administered their respective treatments via intragastric administration $(10 \mathrm{~mL} / \mathrm{kg}$ body weight) for 13 weeks, and an equal volume of distilled water was intragastrically administered to the sham and model groups. XLGB capsules, a traditional Chinese medicine, are popularly used for the treatment of osteoporosis (Dai et al., 2013; Gao et al., 2013). The dosage of XLGB capsules for rats in this study was determined based on the dosage used in clinical trials and calculated using the dose conversion table between human and rats; additionally, the different dosage levels of PTE for rats in this study was determined and calculated according to the $\mathrm{LD}_{50}$ in mice and the daily recommended dose of PTE. The animals were weighed weekly throughout the treatment period. After the treatment period, rats were euthanized by deep ether anesthesia.
Blood samples were obtained for biochemical analyses. The left/right femur, uterus, and vagina were collected, and the samples to be used for histological analysis were maintained in $10 \%$ neutral formaldehyde. The fixed samples were maintained at room temperature for later use.

\section{Biochemical Parameter Analysis of Blood Samples}

The blood was centrifuged at 3,000 rpm for $10 \mathrm{~min}$ at $4^{\circ} \mathrm{C}$ after incubation at room temperature for $2 \mathrm{~h}$. The serum was collected and stored at $-20^{\circ} \mathrm{C}$ for further biochemical analysis. According to the kit instructions, ALP, ACP, calcium, phosphorus, BGP, and $\mathrm{E}_{2}$ levels in rat serum were determined. In addition, IL-1 $\beta$ and IL-6 levels in rat plasma were measured.

\section{Determination of Organ Coefficients}

The left femur, uterus, and vagina were completely separated and weighed. Then, the organ coefficients were calculated as follows: organ coefficient of the wet or dry femur = wet or dry weight of the femur/body weight; organ coefficient of the uterus or vagina $=$ wet weight of the uterus or vagina/body weight.

\section{Detection of Bone Mineral Density and Biomechanical Testing}

The complete left femur of the rat was collected, and muscle and connective tissue was peeled off before analysis. Femoral bone mineral density (BMD) was rapidly determined using dual-energy X-ray absorptiometry (DEXA) according to the manufacturer's instructions (Chunxiao et al., 2017). Briefly, the left femur was scanned, and the femoral BMD value was automatically measured.

The biomechanical properties of the left femur in OVX rats were evaluated by the three-point bending flexural test method (Gao et al., 2013; Chunxiao et al., 2017). Briefly, the femur was placed in a biomechanical testing instrument (Changchun Research Institute for Mechanical Science Co., Ltd, China). The conditions were as follows: stride distance, $20 \mathrm{~mm}$; and loading velocity, $5 \mathrm{~mm} / \mathrm{s}$. The data were recorded with a computer, and the maximum deflection and maximum load were calculated.

\section{Uterus, Vagina, and Femur Histological Analyses}

For the rat uterus, vagina, and right femur histological studies, the fresh tissues were rapidly collected, fixed in 10\% neutral formaldehyde for $72 \mathrm{~h}$, and removed and dehydrated before embedding. The paraffin-embedded tissues were cut into $4-\mu \mathrm{m}$ sections, which were stained with hematoxylin and eosin (H\&E) according to standard techniques as previously described (Wang et al., 2016). The uterus and vagina sections were subsequently observed under a microscope. Static images of the structures of the cortical and trabecular bone were acquired using a medical image analysis system (BI-2000, Taimeng, Chengdu Technology and Market Co., Ltd, Chengdu, China). The cortical bone thickness and trabecular bone area were measured by computeraided software. 


\section{Cell Culture and Maintenance}

RAW264.7 murine macrophages (ATCC, Manassas, VA, United States) were used in this study. Cells were cultured in Dulbecco's modified Eagle's medium (DMEM) supplemented with 10\% fetal bovine serum (FBS) at $37^{\circ} \mathrm{C}$ in a humidified atmosphere with 5\% $\mathrm{CO}_{2}$. DMEM and FBS were obtained from Thermo Fisher Scientific and Biological Industries Israel Beit Haemek Ltd, respectively.

\section{Cytotoxicity Assay}

To evaluate the effect of PTE on the viability of RAW264.7 cells, a cytotoxicity assay was performed using the standard MTT method (Zhang et al., 2016). Briefly, the cells were seeded onto 96-well plates at a density of $3 \times 10^{4}$ cells/well. After overnight incubation, the cells were treated with different concentrations of PTE for $24 \mathrm{~h}$. After incubating the cells with MTT solution for $4 \mathrm{~h}$, the absorbance at $492 \mathrm{~nm}$ was detected using a FlexStation 3 Multi-Mode Microplate Reader (Molecular Devices). Cell viability was expressed as a percentage of the control.

\section{In Vitro Osteoclastogenesis Assay}

To induce osteoclasts, RAW264.7 cells $\left(2 \times 10^{3}\right.$ cells/well $)$ were cultured in the presence of RANKL (50 ng/mL), XLGB (10 $\mu \mathrm{g} / \mathrm{mL})$, and various concentrations of PTE $(20,40$, or $80 \mu \mathrm{g} / \mathrm{mL}$ ). After 6 days, cells were fixed and then stained for TRAP activity according to the manufacturer's protocol. TRAPpositive multinucleated cells with more than five nuclei were counted as mature osteoclasts under a light microscope (Li et al., 2011; Zeng et al., 2016).

\section{Quantitative Real-Time PCR (qRT-PCR) Analysis}

RAW264.7 cells $\left(1.2 \times 10^{5}\right.$ cells/well $)$ were seeded in a 12 -well plate and then treated with RANKL $(50 \mathrm{ng} / \mathrm{mL})$ in the absence or presence of XLGB $(10 \mu \mathrm{g} / \mathrm{mL})$ or PTE $(20$ or $40 \mu \mathrm{g} / \mathrm{mL})$ for $48 \mathrm{~h}$.

Total RNA was extracted by TransZol Up (TransGen Biotech, Beijing, China) according to the manufacturer's protocol. Reverse transcription was performed using the PrimeScript RT Reagent Kit with gDNA Eraser (TaKaRa Bio, Otsu, Japan) according to the manufacturer's protocol. qRT-PCR was performed using SYBR $^{\circledR}$ Premix Ex Taq ${ }^{\text {TM }}$ II (Tli RNaseH Plus, TaKaRa Bio), and the results were determined using a 7900HT Fast RealTime PCR system (Applied Biosystems, Foster City, CA, United States). Data were calculated using the comparative $2^{-\Delta \Delta C T}$ method, and all values were normalized to the mRNA level of the endogenous gene GAPDH (Wang et al., 2016). The primer sequences (Generay Biotech, Shanghai, China) are provided in Table 1.

\section{Protein Preparation and Western Blot Analysis}

RAW264.7 cells $\left(4 \times 10^{5}\right.$ cells/well $)$ were seeded in $60-\mathrm{mm}$ plates and incubated overnight, and they were then treated with RANKL $(50 \mathrm{ng} / \mathrm{mL})$ in the absence or presence of XLGB $(10 \mu \mathrm{g} / \mathrm{mL})$ or PTE $(10,20$, or $40 \mu \mathrm{g} / \mathrm{mL})$ for $48 \mathrm{~h}$.
Western blot analysis was performed as previously described (Cai et al., 2016). Briefly, cell lysates were prepared from cultured cells using RIPA buffer (Solarbio, Beijing, China) according to the manufacturer's protocol. Cell extracts were normalized to determine protein concentration by the BCA method. The proteins were separated by SDS-PAGE and then transferred to PVDF membranes (EMD Millipore Corporation, Merck Life Sciences, KGaA, Darmstadt, Germany). After gentle washing, blocking, and incubation with the primary antibody, the membrane was incubated with an appropriate horseradish peroxidase-conjugated secondary antibody, and the bands were detected using a Pro-light HRP Chemiluminescent Kit (Tiangen Biotech, Beijing, China). The images were acquired with a FluorChem E System (ProteinSimple, Santa Clara, CA, United States).

\section{Statistical Analyses}

All data are presented as the mean \pm the standard errors of the mean (SEM) of the results for the samples in each experimental group or triplicate samples. Differences within groups were statistically analyzed using Student's $t$-test. $P<0.05$ was considered statistically significant. All analyses were performed using SPSS 17.0 (Chicago, IL, United States) and GraphPad Prism 5 (GraphPad Software, Inc., La Jolla, CA, United States).

\section{RESULTS}

\section{PTE Reduced OVX-Induced Body Weight Gain in Rats}

As shown in Table 2, body weight gain with increasing age was observed in our study. The body weight of the model group significantly increased throughout the treatment period compared with that of the sham group $(P<0.01)$ despite the fact that the same amount of food was provided to both groups. A decreasing trend in body weight was not observed in the XLGB group compared with the model group. There were no significant differences between the PTE groups and the model group during the first 5 weeks. However, OVX rats treated with high-dose PTE exhibited rapid body weight decreases at weeks $6,7,8$, and 13 compared with the model group $(P<0.05)$. In addition, the low- and medium-doses of PTE had a tendency to reduce the OVX-induced body weight gain after 6 weeks.

\section{PTE Ameliorated Blood Biochemical Indicators in OVX Rats}

To examine the effects of PTE on blood biochemical markers in OVX rats, serum $E_{2}$, calcium, phosphorus, ALP, BGP, ACP, and plasma IL-1 $\beta$ and IL-6 levels were determined using the appropriate assay kits (Figure 1).

The level of serum $\mathrm{E}_{2}$ in the model group was significantly lower than that in the sham group $(P<0.01)$. Compared with the model group, OVX rats treated with XLGB had moderately increased $\mathrm{E}_{2}$ levels. There were no differences in the $\mathrm{E}_{2}$ level between the low- and medium-dose PTE groups 
TABLE 1 | Primers used in the qRT-PCR study.

\begin{tabular}{|c|c|c|}
\hline Genes & Forward $\left(5^{\prime}-3^{\prime}\right)$ & Reverse $\left(5^{\prime}-3^{\prime}\right)$ \\
\hline GADPH & AACTTGGCATTGTGGAAGG & ACACATTGGGGGTAGGAACA \\
\hline TRAP & GCTGGAAACCATGATCACCT & GAGTTGCCACACAGCATCAC \\
\hline c-Fos & CAAGCGGAGACAGATCAACTTG & ПтССПТСTCПTCAGCAGATTGG \\
\hline c-Src & CCAGGCTGAGGAGTGGTACT & CAGCTTGCGGATCTTGTAGT \\
\hline$\beta 3$-Intergrin & TGACATCGAGCAGGTGAAAG & GAGTAGCAAGGCCAATGAGC \\
\hline Cathepsin K & CTTCCAATACGTGCAGCAGA & TCTTCAGGGCTाTCTCGTTC \\
\hline MMP-9 & CGTCGTGATCCCCACTTACT & AACACACAGGGTTGCCTTC \\
\hline NFATc1 & TGGAGAAGCAGAGCACAGAC & GCGGAAAGGTGGTATCTCAA \\
\hline
\end{tabular}

TABLE 2 | Effect of Pu-erh tea extract (PTE) on body weight $(\mathrm{g})$ in OVX rats ${ }^{\#}$.

\begin{tabular}{|c|c|c|c|c|c|c|}
\hline Time (week) & Sham & Model & XLGB & Low-dose & Medium-dose & High-dose \\
\hline 0 & $254.0 \pm 17.5^{b}$ & $278.3 \pm 22.2$ & $279.9 \pm 19.9$ & $286.9 \pm 24.1$ & $283.9 \pm 26.5$ & $280.3 \pm 25.0$ \\
\hline 1 & $260.3 \pm 18.7^{b}$ & $290.0 \pm 32.5$ & $298.0 \pm 25.6$ & $299.1 \pm 26.9$ & $296.0 \pm 26.0$ & $288.2 \pm 31.2$ \\
\hline 2 & $263.3 \pm 18.9^{c}$ & $295.8 \pm 21.2$ & $304.4 \pm 29.6$ & $307.6 \pm 29.1$ & $300.9 \pm 35.0$ & $291.6 \pm 29.6$ \\
\hline 3 & $268.6 \pm 17.6^{c}$ & $311.5 \pm 23.6$ & $318.0 \pm 27.9$ & $312.9 \pm 32.6$ & $317.7 \pm 44.0$ & $301.9 \pm 28.0$ \\
\hline 4 & $271.8 \pm 17.8^{\mathrm{c}}$ & $317.5 \pm 22.6$ & $322.2 \pm 27.3$ & $315.6 \pm 35.7$ & $319.1 \pm 47.9$ & $301.9 \pm 29.1$ \\
\hline 5 & $275.1 \pm 16.1^{c}$ & $327.5 \pm 17.2$ & $331.9 \pm 29.1$ & $336.1 \pm 30.1$ & $325.8 \pm 38.8$ & $311.8 \pm 28.5$ \\
\hline 6 & $270.3 \pm 14.2^{\mathrm{C}}$ & $335.9 \pm 14.8$ & $331.0 \pm 29.6$ & $327.4 \pm 33.5$ & $325.7 \pm 36.9$ & $312.3 \pm 27.0^{a}$ \\
\hline 7 & $278.6 \pm 15.6^{c}$ & $343.7 \pm 15.2$ & $336.4 \pm 26.4$ & $333.3 \pm 34.0$ & $336.0 \pm 35.3$ & $318.9 \pm 36.6^{b}$ \\
\hline 8 & $283.1 \pm 24.3^{\mathrm{C}}$ & $353.1 \pm 16.6$ & $338.7 \pm 31.1$ & $341.5 \pm 36.0$ & $343.0 \pm 39.1$ & $328.4 \pm 29.5^{a}$ \\
\hline 9 & $281.0 \pm 24.3^{\mathrm{C}}$ & $353.6 \pm 15.3$ & $349.9 \pm 28.3$ & $345.4 \pm 36.3$ & $348.8 \pm 38.7$ & $335.6 \pm 30.6$ \\
\hline 10 & $284.9 \pm 15.5^{\mathrm{c}}$ & $351.3 \pm 18.2$ & $351.4 \pm 28.4$ & $351.3 \pm 33.2$ & $348.0 \pm 40.6$ & $332.6 \pm 31.2$ \\
\hline 11 & $298.6 \pm 16.3^{c}$ & $352.8 \pm 32.7$ & $362.5 \pm 30.8$ & $352.8 \pm 34.0$ & $351.5 \pm 48.2$ & $340.8 \pm 31.4$ \\
\hline 12 & $296.5 \pm 19.7^{\mathrm{c}}$ & $365.9 \pm 19.2$ & $366.1 \pm 30.8$ & $368.5 \pm 36.0$ & $363.4 \pm 34.8$ & $348.3 \pm 33.2$ \\
\hline 13 & $303.0 \pm 20.8^{c}$ & $367.0 \pm 17.7$ & $366.7 \pm 31.1$ & $370.6 \pm 36.2$ & $363.9 \pm 42.0$ & $345.4 \pm 33.1^{a}$ \\
\hline
\end{tabular}

${ }^{\#}$ All data are presented as the mean \pm SEM $(n=12-14) .{ }^{a} P<0.05,{ }^{b} P<0.01$, and ${ }^{\mathrm{c}} P<0.001$ versus the model group.

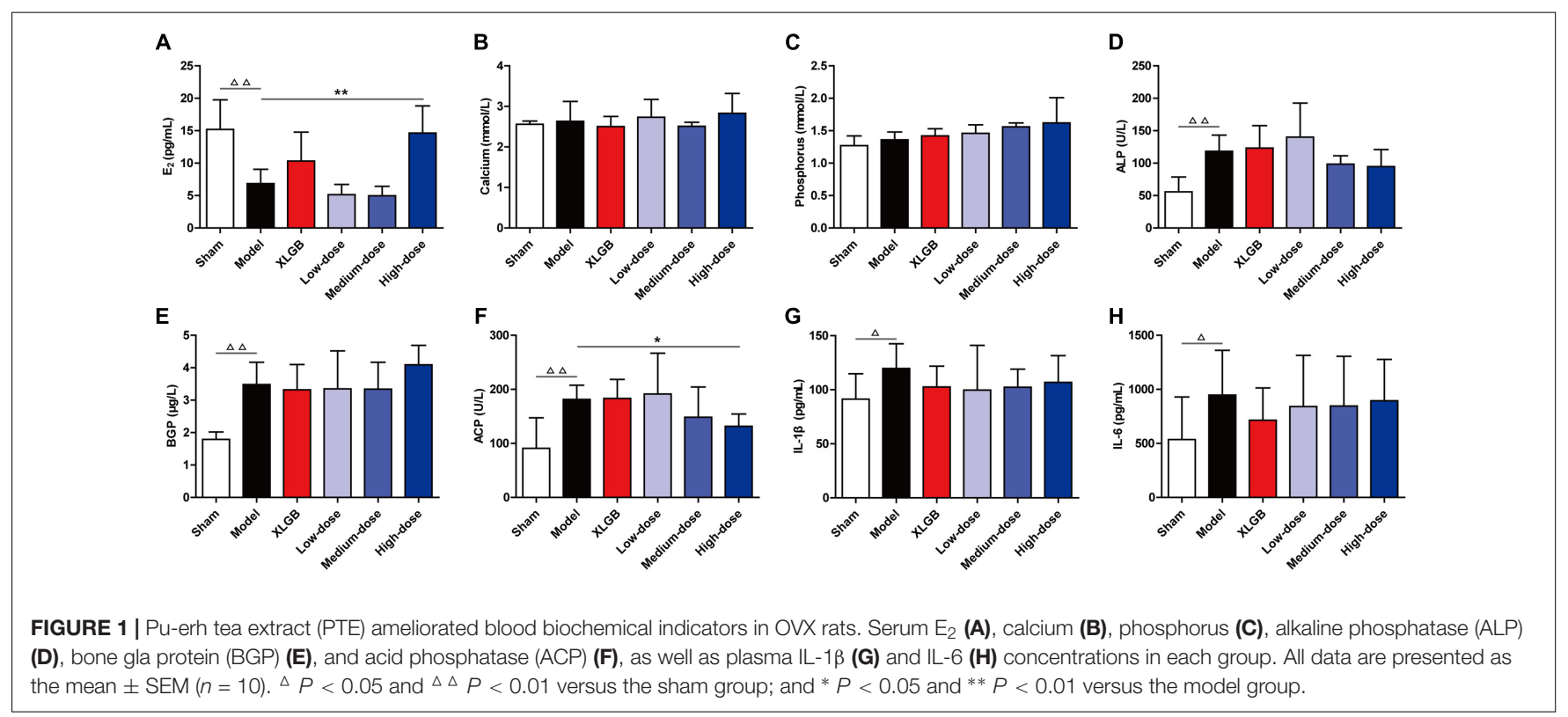

and the model group. However, treatment of OVX rats with PTE at a high-dose significantly increased $E_{2}$ levels compared with those in the model group $(P<0.01$; Figure $1 \mathrm{~A})$.
These findings suggested that $\mathrm{E}_{2}$ levels in OVX rats could be significantly improved and could reach normal levels with highdose PTE treatment. Additionally, high-dose PTE was more 
effective than the drug XLGB in elevating $\mathrm{E}_{2}$ levels in OVX rats.

There were no differences in calcium and phosphorus levels between the model and sham groups (Figures 1B,C). Differences in calcium levels were not observed between the XLGB and model groups. OVX rats treated with PTE at low- and high-doses appeared to have increased calcium levels compared with the model group, but the changes were not significant (Figure 1B). Similarly, differences were not observed in phosphorus levels between the XLGB and model groups. Treatment of OVX rats with PTE increased phosphorus levels in a dose-dependent manner compared with the model group (Figure 1C). These results indicated that treatment of OVX rats with PTE could maintain calcium and phosphorus homeostasis in these animals, although significant differences were not observed between the different groups.

Additionally, we evaluated the serum biomarkers of bone formation (ALP and BGP) and bone resorption (ACP) (Figures 1D-F). These parameters in the model group were significantly increased compared with those in the sham group $(P<0.01)$. OVX rats treated with PTE at medium- and high-doses appeared to have lower ALP levels than did those in the model group (Figure 1D). In OVX rats treated with XLGB and in OVX rats treated with PTE at low- and medium-doses, BGP levels were lower than those in the model group (Figure 1E). Notably, treatment with PTE at a high-dose significantly prevented OVX-induced increases in the ACP level $(P<0.05$; Figure 1F). Bone resorption is associated with many cytokines, including IL-1 $\beta$ and IL-6. There were significant differences in IL-1 $\beta$ and IL-6 levels between the model and sham groups $(P<0.05$; Figures $\mathbf{1 G}, \mathbf{H})$. We detected lower levels of IL- $1 \beta$ and IL- 6 in the plasma of OVX rats with XLGB or PTE treatment at any dose than in the model group. These results revealed that PTE could mildly improve bone homeostasis in OVX rats.

\section{Effects of PTE on the Femur, Uterus, and Vagina Organ Coefficients in OVX Rats}

To further investigate the effects of PTE on osteoporosis, the femur, uterus, and vagina organ coefficients in OVX rats were obtained (Figures 2A-D). Compared with the sham group, the organ coefficients of the femur, uterus, and vagina in the
A

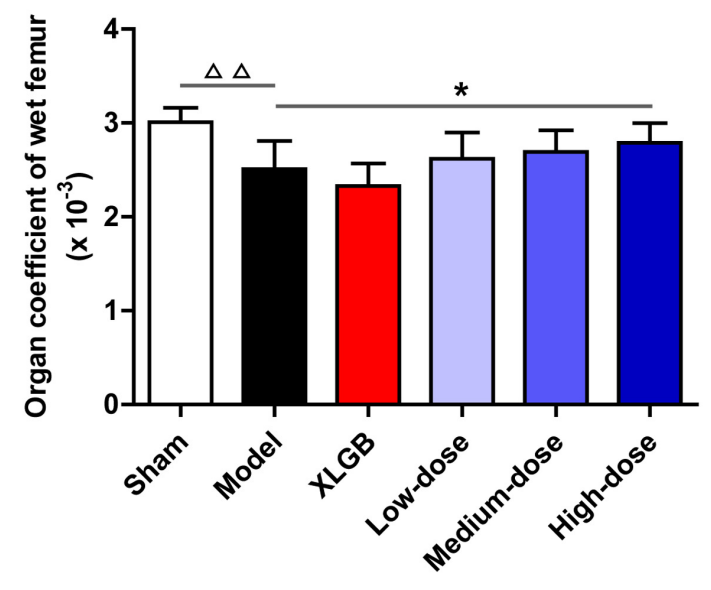

C

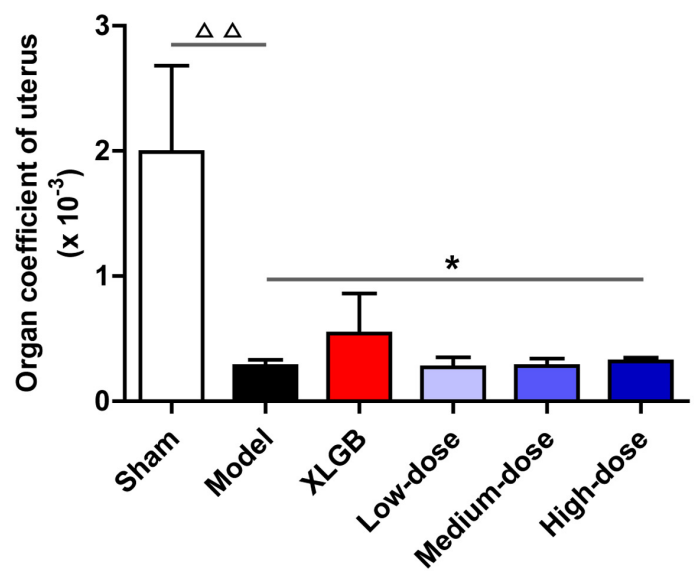

B

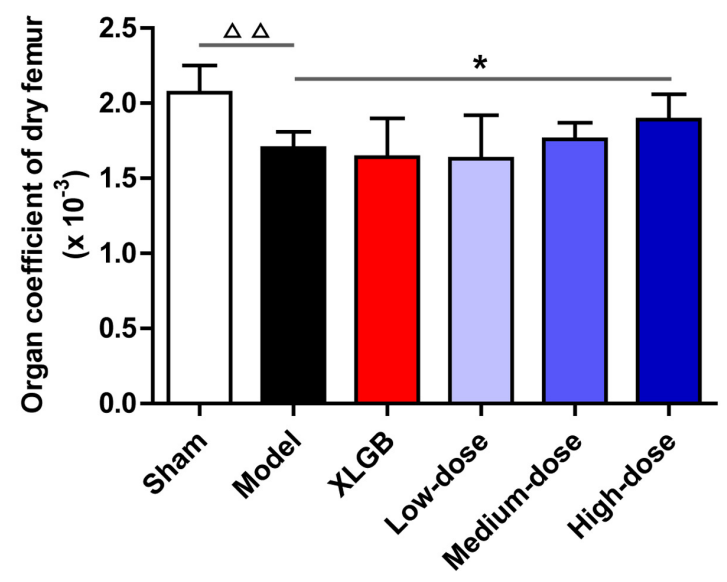

D

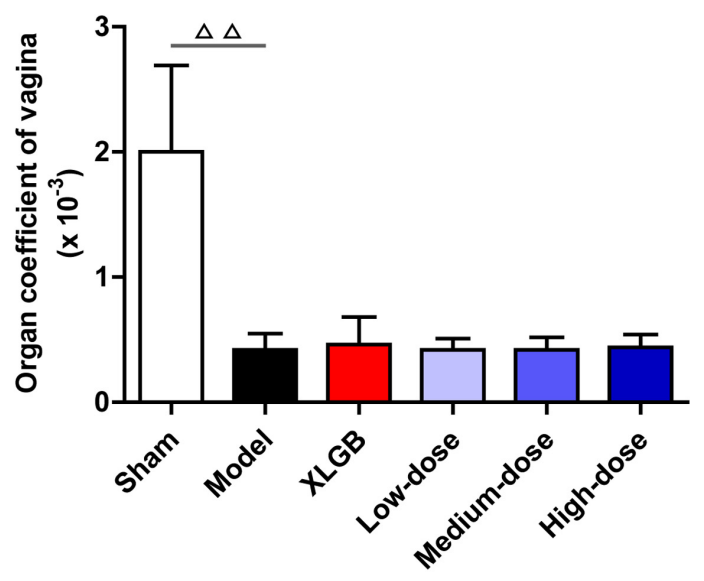

FIGURE 2 | Pu-erh tea extract improved organ coefficients in OVX rats. Organ coefficients of the wet femur (A), dry femur (B), uterus (C), and vagina (D) in each group. All data are presented as the mean $\pm \operatorname{SEM}(n=10)$. $\Delta \Delta P<0.01$ versus the sham group; and ${ }^{*} P<0.05$ versus the model group. 
model group all significantly decreased $(P<0.01)$. Interestingly, compared with the model group, the OVX rats treated with PTE increased the wet femur organ coefficient in a dose-dependent manner, and the high-dose PTE treatment led to a significant difference $(P<0.05$; Figure 2A). A similar pattern was observed in the dry femur organ coefficient between the high- and medium-dose PTE groups and the model group $(P<0.05$ at the high-dose; Figure 2B). However, no difference was observed in the organ coefficient of the uterus between almost all groups of OVX rats that underwent different treatments, except that a significant increase was observed in the high-dose PTE group compared with the model group $(P<0.05$; Figure $2 \mathrm{C})$. There were no significant differences in the organ coefficients of the vagina between the different treatment groups (Figure 2D). In addition, to investigate whether PTE protected the uterus and vagina from OVX-induced atrophy, we performed histological analyses of both tissues. H\&E staining showed that PTE treatment ameliorated uterus and vagina atrophy in OVX rats, but this effect was not obvious.

\section{PTE Improved Femoral BMD, Biomechanical Properties, and Bone Microarchitecture in OVX Rats}

To further identify the osteoprotective effect of PTE, the $\mathrm{BMD}$, biomechanical properties, and bone microarchitecture of the femur in OVX rats were determined (Figure 3). The femoral BMD of the OVX rats significantly decreased to $0.297 \pm 0.013 \mathrm{~g} / \mathrm{cm}^{2}$, compared with $0.312 \pm 0.025 \mathrm{~g} / \mathrm{cm}^{2}$ in the sham rats $(P<0.05)$, indicating that ovariectomy decreased the rat femoral BMD by $4.8 \%$ after 15 weeks. Compared with the model group, the decreased femoral BMD was significantly reversed $(P<0.05)$ by XLGB administration. Compared with the model group, OVX rats treated with PTE seemed to increase femoral BMD in a dosedependent manner, but significant differences were not observed (Figure 3A).

Regarding the femoral biomechanical properties (maximum deflection and maximum load), there were no significant differences between the model and sham groups (Figures 3B,C). Compared with the model group, OVX rats treated with XLGB and PTE showed elevated maximum deflection, but no significant differences were observed (Figure 3B). However, compared with the model group, OVX rats treated with PTE exhibited an increased maximum load, which was significant with the lowdose PTE treatment $(P<0.05$; Figure 3C).

The above results suggest that PTE treatment could improve femoral BMD and biomechanical properties in OVX rats. To substantiate this finding further, we measured the cortical and trabecular bone microarchitectures and analyzed the cortical bone and trabecular bone parameters in the femur. Unsurprisingly, the cortical bone thickness in the model group was significantly decreased compared with that in the sham group $(P<0.05)$. Interestingly, PTE treatment effectively increased the cortical bone thickness compared with that in the model group, and the medium- and high-dose PTE groups exhibited significant differences $(P<0.05$; Figures 3D,E). Similarly, the trabecular bone area in the model group was significantly decreased compared with that in the sham group $(P<0.01)$, whereas XLGB and PTE treatments significantly reversed trabecular bone loss in OVX rats $(P<0.05$ or $P<0.01$; Figures 3F,G). Notably, the treatment of OVX rats with PTE significantly increased the trabecular bone area in a dose-dependent manner compared with the model group $(P<0.01)$. These results suggested that PTE had a protective effect on the bone quality of OVX rats.

\section{PTE Inhibited Osteoclast Differentiation in the RAW264.7 Cell Line}

To investigate the effect of PTE on osteoclastogenesis, a standard in vitro osteoclast differentiation model was used. We incubated RANKL-treated RAW264.7 cells with XLGB or various concentrations of PTE and then evaluated the formation of osteoclasts using TRAP staining. TRAP-positive multinucleated cells were formed in response to RANKL stimulation, and XLGB or PTE treatment significantly reduced the number of RANKLinduced osteoclasts $(P<0.05$ or $P<0.01$; Figures 4A,B). To determine whether the inhibition of osteoclastogenesis by PTE is due to cytotoxicity, we further examined the cytotoxicity of PTE in RAW264.7 cells using the MTT assay (Figure 4C). As expected, PTE did not have a cytotoxic effect on osteoclast precursor cells at the concentrations used in this study. These results suggested that PTE inhibited RANKL-induced osteoclast differentiation in RAW264.7 cells.

\section{PTE Suppressed RANKL-Induced Osteoclast-Specific Gene and Protein Expression}

To further examine the effect of PTE on osteoclast differentiation in RAW264.7 cells, we evaluated the expression of RANKLinduced osteoclast-specific gene markers at the mRNA level in the absence or presence of XLGB $(10 \mu \mathrm{g} / \mathrm{mL})$ or PTE (20 or $40 \mu \mathrm{g} / \mathrm{mL})$. As shown in Figure 5, the mRNA expression levels of TRAP, c-Fos, c-Src, $\beta 3$-Integrin, cathepsin $\mathrm{K}$, matrix metalloproteinase-9 (MMP-9), and NFATc1 were significantly suppressed by XLGB or PTE during osteoclastogenesis $(P<0.001)$. Furthermore, we detected the expression of key osteoclast-specific protein markers in the absence or presence of XLGB or various concentrations of PTE. We found that XLGB or PTE treatment could significantly down-regulate the protein expression of NFATc1, c-Src, c-Fos, and cathepsin K during osteoclastogenesis $(P<0.05, P<0.01$, or $P<0.001$; Figure 6), which was consistent with the results of the qRT-PCR analysis. These results strongly revealed that PTE could inhibit osteoclastogenesis.

\section{DISCUSSION}

$\mathrm{Pu}$-erh tea, a postfermented tea that was originally grown and produced in southern Yunnan, China, is widely consumed in southeastern Asia because it has been confirmed to possess many health benefits, including weight loss, lipid metabolism regulation, cardiovascular disease alleviation, and 
A

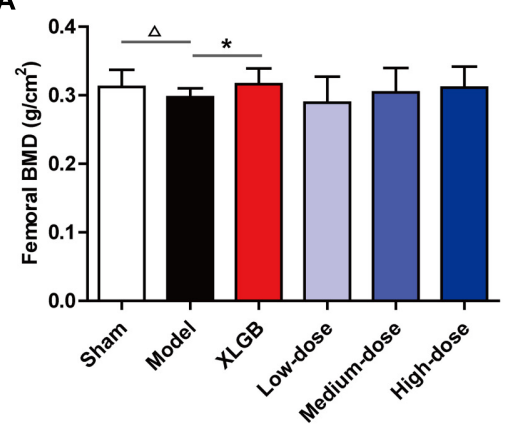

D
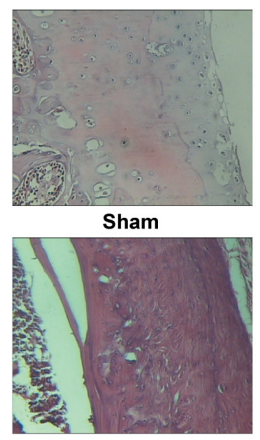

Low-dose

F

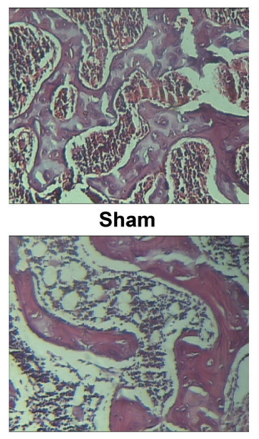

Low-dose

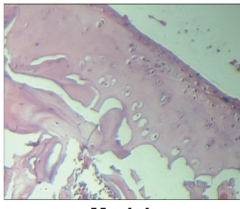

Model

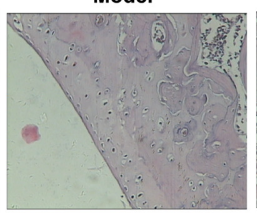

Medium-dose

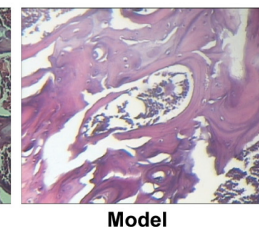

Model

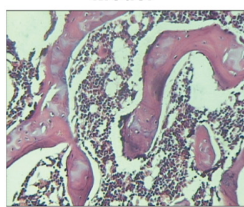

Medium-dose
B

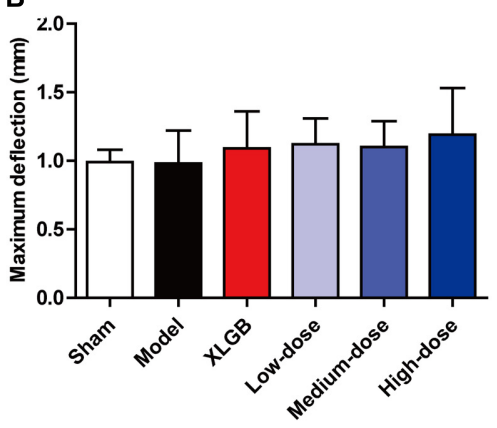

C

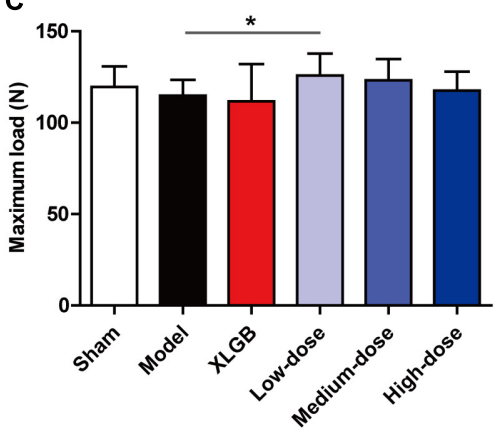

E
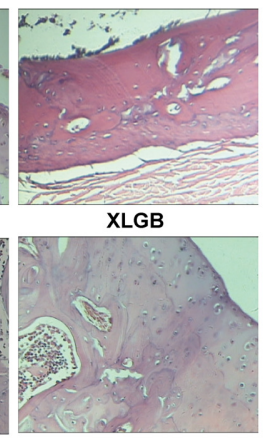

High-dose

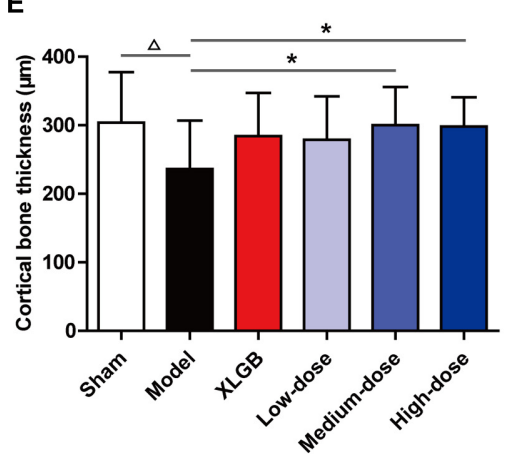

G
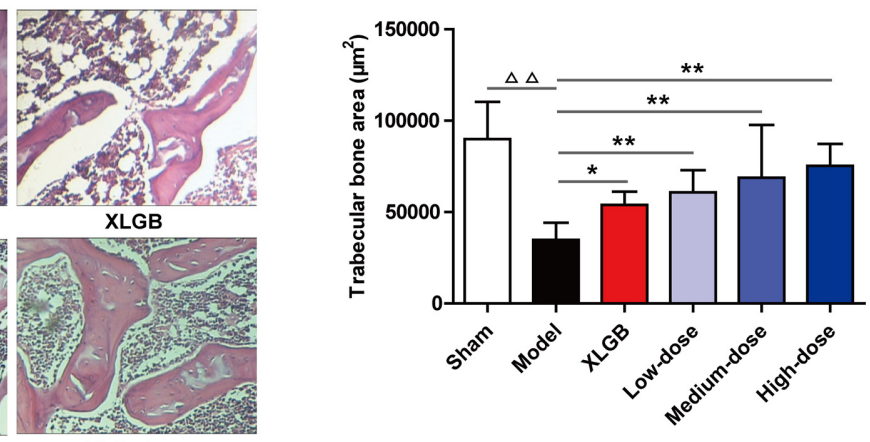

High-dose

FIGURE 3 | Pu-erh tea extract preserved bone quality in OVX rats. Femoral bone mineral density (BMD) (A), maximum deflection (B), and maximum load (C) in each group. Cortical bone tissue was stained with hematoxylin and eosin (H\&E) (D), and cortical bone thickness was calculated (E); and trabecular bone tissue was stained with $\mathrm{H} \& \mathrm{E}(\mathbf{F})$, and the trabecular bone area was calculated (G). Representative images were acquired using a medical image analysis system, and the original magnification was $\times 400$. All data are presented as the mean \pm SEM $(n=10) . \Delta P<0.05$ and $\Delta \Delta P<0.01$ versus the sham group; and $* P<0.05$ and ** $P<0.01$ versus the model group.

nervous system protection (Hou et al., 2009; Kubota et al., 2011; Zeng et al., 2015; Cai et al., 2016; Li et al., 2016). Numerous studies have demonstrated that tea drinking is associated with positive effects on bone health, especially in elderly women (Hegarty et al., 2000; Devine et al., 2007; Conforti et al., 2012; Faucher, 2012; Huang and Tang, 2016). However, the potential bone protective effects of $\mathrm{Pu}$-erh tea remain unclear. The OVX rat is the optimal animal model for the study of postmenopausal osteoporosis in women and is recommended by the US Food and Drug Administration and the World Health Organization (Hartke, 1999; Pan et al.,
2007). In the present study, we systematically investigated the effects of PTE on ovariectomy-induced osteoporosis in rats and further investigated the in vitro effects of PTE on osteoclastic activities using RAW264.7 cells. Intriguingly, our results indicated that PTE could ameliorate osteoporosis in vivo and in vitro.

Unambiguously, body weight gain is a common phenomenon observed in OVX rats, and postmenopausal women are associated with a tendency to gain weight (Lee et al., 2016). In this study, the body weight of the model group was significantly higher than that of the sham group $(P<0.01$; Table 2$)$. At 


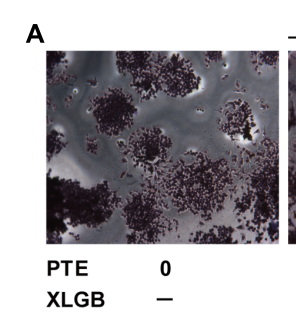

B

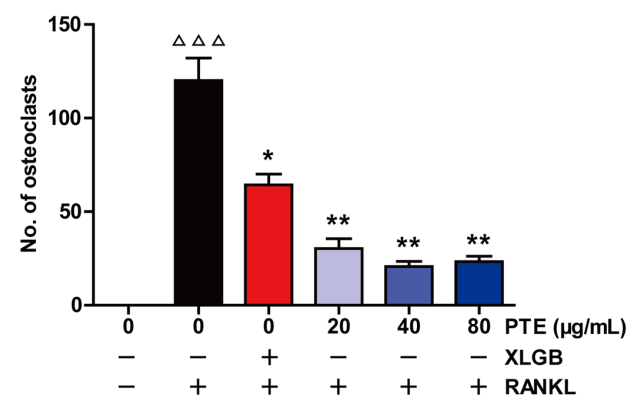

RANKL $50 \mathrm{ng} / \mathrm{mL}$

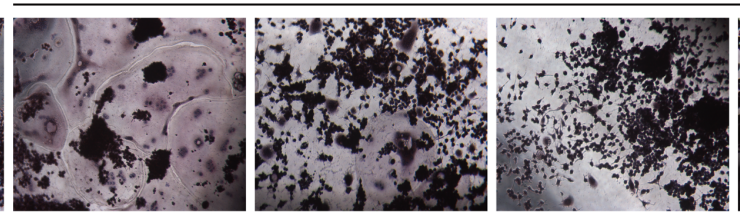

$20 \mu \mathrm{g} / \mathrm{mL}$

-

C

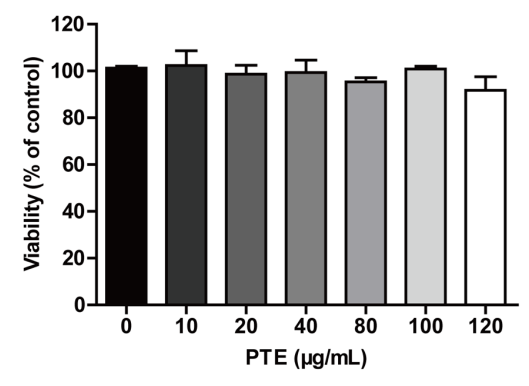

FIGURE 4 | Pu-erh tea extract inhibited osteoclast differentiation in the RAW264.7 cells. RAW264.7 cells were cultured for 6 days with RANKL (50 ng/mL) in the presence of Xian-Ling-Gu-Bao (XLGB) $(10 \mu \mathrm{g} / \mathrm{mL})$ or the indicated concentrations of PTE and then stained for TRAP (A). TRAP-positive multinucleated cells with more than five nuclei were considered mature osteoclasts, as observed under a light microscope (B). The effect of PTE on the viability of RAW264.7 cells as determined by the MTT assay (C). Representative images were acquired under a light microscope (magnification $\times 200)$. Values are shown as the mean \pm SEM of three independent experiments. $\Delta \Delta \Delta P<0.001$ versus the control; and $* P<0.05$ and $* * P<0.01$ versus only RANKL-treated cells.
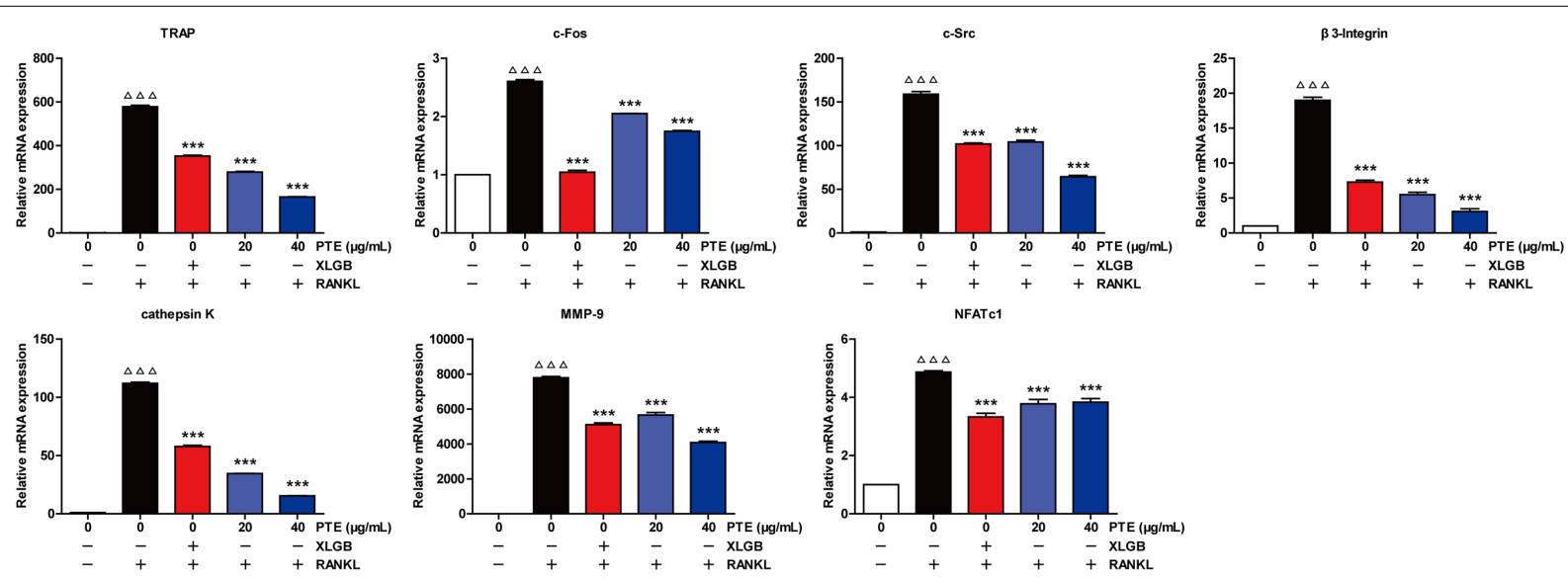

FIGURE 5 | Pu-erh tea extract suppressed the RANKL-induced mRNA expression of osteoclast-specific genes. Values are shown as the mean \pm SEM of three independent experiments. $\Delta \Delta \Delta P<0.001$ versus the control; and ${ }^{* * *} P<0.001$ versus only RANKL-treated cells.

the beginning of the treatment, PTE treatment did not show an effect on lowering body weight. However, PTE reduced OVX-induced body weight gain in rats only after 6 weeks of treatment, suggesting that long-term tea drinking has a positive effect on weight loss, which is consistent with the results of previous studies (Zou et al., 2012). Notably, osteoporosis results from an imbalance related to faster bone resorption than bone formation. Therefore, we further investigated the biochemical markers for bone formation and resorption in blood samples (Figure 1). Menopause is closely associated with estrogen deficiency, which leads to decreased calcium absorption capacity in the small intestines that may accelerate the pathogenesis of osteoporosis (Das et al., 2013). In our study, we observed that the administration of high-dose PTE significantly increased serum $\mathrm{E}_{2}$ levels compared with those in the model group $(P<0.01)$. Previous studies have reported that high caffeine intake is a risk factor for increased calcium excretion in postmenopausal women (Macedo et al., 2015; Costa et al., 2016). Although Pu-erh tea has a high caffeine content (Huang et al., 2014), PTE could maintain calcium and phosphorus homeostasis in OVX rats. Our previous study demonstrated that the absorption of caffeine can be partly inhibited by OTP in Pu-erh tea (Huang et al., 2014), which may explain why PTE did not accelerate calcium and phosphorus loss. Moreover, PTE could lower ALP, BGP, ACP, IL-1 $\beta$, and IL-6 levels 

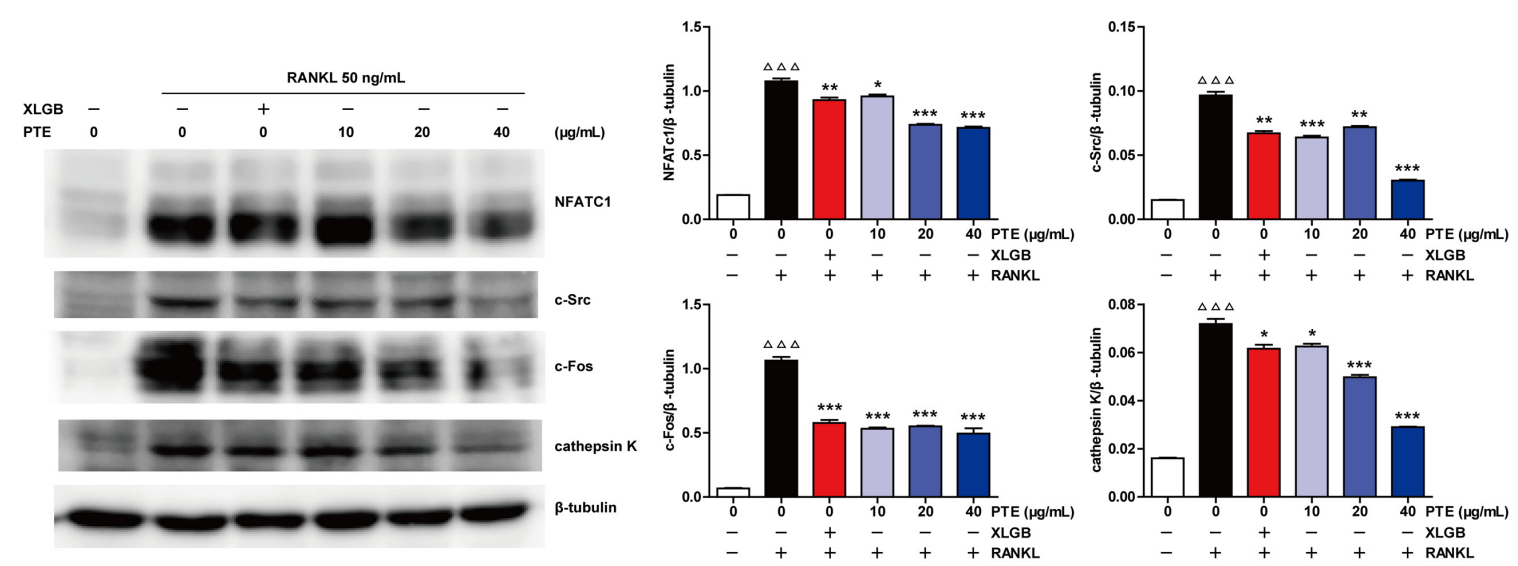

FIGURE 6 | Pu-erh tea extract inhibited the expression of the RANKL-induced osteoclast-specific proteins. NFATc1, c-Src, c-Fos, cathepsin K, and $\beta$-tubulin levels were detected by western blotting, and the results were quantified using AlphaView software. Representative images are displayed. Values are shown as the mean \pm SEM of three independent experiments. $\Delta \Delta \Delta P<0.001$ versus the control; and $* P<0.05$, ${ }^{* *} P<0.01$, and ${ }^{* * *} P<0.001$ versus only RANKL-treated cells.

in OVX rats depending on the dosage administered. Overall, PTE can ameliorate these blood biochemical indicators in OVX rats and prevent the deterioration of osteoporosis.

Importantly, in vivo treatment with PTE, especially at the high-dose, effectively increased the organ coefficients of the femur, uterus, and vagina in OVX rats (Figure 2), suggesting that PTE has protective effects on femoral, uterine, and vaginal tissues. As previously described, osteoporosis is a type of systemic skeletal disease that is characterized by bone loss and microstructure degradation of bone tissue accompanied by increased bone fragility and fracture susceptibility (Tabatabaei-Malazy et al., 2017). Femoral BMD, biomechanical properties (maximum deflection and maximum load), and bone microarchitecture are commonly used to evaluate bone quality. Our results showed that PTE treatment improved the femoral BMD and biomechanical parameters in OVX rats compared with the model group (Figures 3A-C). Treatment with PTE ameliorated bone microarchitecture in OVX rats due to increased cortical bone thickness and trabecular bone area in the femur (Figures 3D-G). These results implied that PTE could exert protective effects on bone quality. Previous studies have reported that there are many chemical components contained in the fermented $\mathrm{Pu}$-erh tea, including flavan-3-ols and their derivatives, flavones and their derivatives, other phenolic compounds, alkaloids and their derivatives, and other compounds and trace elements (Lv et al., 2013). After the post-fermentation process, the caffeine, polysaccharide, and tea pigment (theaflavin, thearubigin, and theabrownin) levels were substantially increased in Pu-erh tea (Zhao L. et al., 2011; Lv et al., 2013). Notably, tea polyphenols have undergone a series of oxidative, condensing and degradative chemical processes to form oxidized tea polyphenols, which have many positive effects on human health (Lv et al., 2013; Huang et al., 2014; Shan et al., 2016; Wang et al., 2016; Lu et al., 2017). Because of the extraordinary complexity of its components, the bioactive constituents of Pu-erh tea are largely unknown. Therefore, further study is urgently required to investigate the bioactive components responsible for the anti-osteoporotic effect of Pu-erh Tea.

Evidence from in vitro studies indicated that the bioactive constituents found in tea may benefit bone health by enhancing osteoblastogenesis and suppressing osteoclastogenesis through the regulation of related signaling pathways (Nakashima and Haneji, 2013; Shen et al., 2013; Visagie et al., 2015). The RAW 264.7 cell line has proven to be suitable for in vitro studies of RANKL-induced osteoclast formation (Collin-Osdoby and Osdoby, 2012). Interestingly, our in vitro studies showed that PTE treatment in the differentiation cell model significantly inhibited osteoclast differentiation without cytotoxic effects (Figure 4). It is well known that osteoclast differentiation is directed by the expression of many related marker genes, such as NFATc1, TRAP, c-Fos, c-Src, $\beta 3$-Integrin, cathepsin K, and MMP-9, most of which are regulated by NFATc1 (Li et al., 2011; Zeng et al., 2016). In this study, PTE treatment was able to effectively suppress the expression of osteoclast-specific genes (Figure 5) and proteins (Figure 6). However, future studies should investigate the precise mechanisms of PTE or its bioactive constituents in the inhibition of osteoclastogenesis.

\section{CONCLUSION}

Taken together, we demonstrated that PTE can ameliorate ovariectomy-induced osteoporosis in rats and suppress osteoclastogenesis in vitro, suggesting that PTE could be used as a promising agent in preventing and treating osteoporosis.

\section{AUTHOR CONTRIBUTIONS}

JS, XW, and HX conceived and designed the experiments. $\mathrm{HX}, \mathrm{TL}, \mathrm{SD}, \mathrm{DY}$, and XC performed the experiments. HX, TL, CX, and XW analyzed the data. JS and XW contributed reagents/materials/analysis tools. $\mathrm{HX}, \mathrm{TL}$, and XW wrote the manuscript. All authors read and approved the final manuscript. 


\section{FUNDING}

This work was financially supported by grants from the Natural Science Foundation of China (No. 31460392), the Natural Science Foundation of Yunnan Province (No. 2014FB180), and the Scholarship for Young Academic and Technological Leaders of Yunnan Province (No. 2015HB037).

\section{REFERENCES}

Boyle, W. J., Simonet, W. S., and Lacey, D. L. (2003). Osteoclast differentiation and activation. Nature 423, 337-342. doi: 10.1038/nature01658

Byun, M. R., Sung, M. K., Kim, A. R., Lee, C. H., Jang, E. J., Jeong, M. G., et al. (2014). (-)-Epicatechin gallate (ECG) stimulates osteoblast differentiation via Runt-related transcription factor 2 (RUNX2) and transcriptional coactivator with PDZ-binding motif (TAZ)-mediated transcriptional activation. J. Biol. Chem. 289, 9926-9935. doi: 10.1074/jbc.M113.522870

Cai, X., Fang, C., Hayashi, S., Hao, S., Zhao, M., Tsutsui, H., et al. (2016). Puerh tea extract ameliorates high-fat diet-induced nonalcoholic steatohepatitis and insulin resistance by modulating hepatic IL-6/STAT3 signaling in mice. J. Gastroenterol. 51, 819-829. doi: 10.1007/s00535-015-1154-0

Chunxiao, W., Chengying, G., Liang, J., Xiaoming, S., Feng, G., Junting, Y., et al. (2017). Pharmacological effects of a recombinant hPTH(1-34) derived peptide on ovariectomized rats. Eur. J. Pharmacol. 794, 193-200. doi: 10.1016/j.ejphar. 2016.11.034

Collin-Osdoby, P., and Osdoby, P. (2012). RANKL-mediated osteoclast formation from murine RAW 264.7 cells. Methods Mol. Biol. 816, 187-202. doi: 10.1007/ 978-1-61779-415-5_13

Conforti, A. S., Gallo, M. E., and Saravi, F. D. (2012). Yerba Mate (Ilex paraguariensis) consumption is associated with higher bone mineral density in postmenopausal women. Bone 50, 9-13. doi: 10.1016/j.bone.2011.08.029

Costa, A. L., da Silva, M. A., Brito, L. M., Nascimento, A. C., do Carmo Lacerda Barbosa, M., Batista, J. E., et al. (2016). Osteoporosis in primary care: an opportunity to approach risk factors. Rev. Bras. Reumatol. Engl. Ed. 56, 111-116. doi: 10.1016/j.rbre.2015.07.014

Dai, Y., Tu, F. J., Yao, Z. H., Ding, B., Xu, W., Qiu, X. H., et al. (2013). Rapid identification of chemical constituents in traditional Chinese medicine fufang preparation xianling gubao capsule by LC-linear ion trap/Orbitrap mass spectrometry. Am. J. Chin. Med. 41, 1181-1198. doi: 10.1142/ S0192415X13500808

Das, A. S., Banerjee, M., Das, D., Mukherjee, S., and Mitra, C. (2013). Black tea may be a prospective adjunct for calcium supplementation to prevent early menopausal bone loss in a rat model of osteoporosis. J. Osteoporos. 2013:760586. doi: $10.1155 / 2013 / 760586$

Das, A. S., Das, D., Mukherjee, M., Mukherjee, S., and Mitra, C. (2005). Phytoestrogenic effects of black tea extract (Camellia sinensis) in an oophorectomized rat (Rattus norvegicus) model of osteoporosis. Life Sci. 77, 3049-3057. doi: 10.1016/j.lfs.2005.02.035

Das, A. S., Mukherjee, M., and Mitra, C. (2004). Evidence for a prospective anti-osteoporosis effect of black tea (Camellia Sinensis) extract in a bilaterally ovariectomized rat model. Asia Pac. J. Clin. Nutr. 13, 210-216.

Devine, A., Hodgson, J. M., Dick, I. M., and Prince, R. L. (2007). Tea drinking is associated with benefits on bone density in older women. Am. J. Clin. Nutr. 86, 1243-1247.

Faucher, M. A. (2012). Drink tea for bone health but not too much. J. Midwifery Womens Health 57, 421-422. doi: 10.1111/j.1542-2011.2012. 00195_3.x

Gao, Z. M., Yang, L., Huang, F., Xiong, A. H., Zhou, N., Zhou, L., et al. (2013). Effects of different extracts of kanggushu on osteoporosis in model rats and the underlying mechanisms. Chin. J. Integr. Med. 19, 844-852. doi: 10.1007/s11655012-1190-z

Hartke, J. R. (1999). Preclinical development of agents for the treatment of osteoporosis. Toxicol. Pathol. 27, 143-147. doi: 10.1177/019262339902700126

Hegarty, V. M., May, H. M., and Khaw, K. T. (2000). Tea drinking and bone mineral density in older women. Am. J. Clin. Nutr. 71, 1003-1007.

\section{ACKNOWLEDGMENTS}

We are grateful to professor Yingli Chen (Jilin Academy of Traditional Chinese Medicine, China) for providing excellent technical support in animal experiments. We also thank Dr. Yewei Huang for his editorial assistance with this manuscript.

Hou, Y., Shao, W., Xiao, R., Xu, K., Ma, Z., Johnstone, B. H., et al. (2009). Pu-erh tea aqueous extracts lower atherosclerotic risk factors in a rat hyperlipidemia model. Exp. Gerontol. 44, 434-439. doi: 10.1016/j.exger.2009.03.007

Huang, C., and Tang, R. (2016). Tea drinking habits and osteoporotic hip/femur fractures: a case-control study. Pak. J. Med. Sci. 32, 408-412. doi: 10.12669/pjms. 322.9092

Huang, Y. W., Xu, H. H., Wang, S. M., Zhao, Y., Huang, Y. M., Li, R. B., et al. (2014). Absorption of caffeine in fermented Pu-er tea is inhibited in mice. Food Funct. 5, 1520-1528. doi: 10.1039/c4fo00051j

Kleerekoper, M. (2006). Osteoporosis prevention and therapy: preserving and building strength through bone quality. Osteoporos. Int. 17, 1707-1715. doi: 10.1007/s00198-006-0187-x

Kubota, K., Sumi, S., Tojo, H., Sumi-Inoue, Y., I-Chin, H., Oi, Y., et al. (2011). Improvements of mean body mass index and body weight in preobese and overweight Japanese adults with black Chinese tea (Pu-Erh) water extract. Nutr. Res. 31, 421-428. doi: 10.1016/j.nutres.2011.05.004

Lee, M. Y., Kim, H. Y., Singh, D., Yeo, S. H., Baek, S. Y., Park, Y. K., et al. (2016). Metabolite profiling reveals the effect of dietary Rubus coreanus vinegar on ovariectomy-induced osteoporosis in a rat model. Molecules 21:149. doi: 10.3390/molecules21020149

Li, C., Chai, S., Ju, Y., Hou, L., Zhao, H., Ma, W., et al. (2016). Pu-erh tea protects the nervous system by inhibiting the expression of metabotropic glutamate receptor 5. Mol. Neurobiol. doi: 10.1007/s12035-016-0064-3 [Epub ahead of print].

Li, C., Yang, Z., Li, Z., Ma, Y., Zhang, L., Zheng, C., et al. (2011). Maslinic acid suppresses osteoclastogenesis and prevents ovariectomy-induced bone loss by regulating RANKL-mediated NF- $\mathrm{BB}$ and MAPK signaling pathways. J. Bone Miner. Res. 26, 644-656. doi: 10.1002/jbmr.242

Li, G., Thabane, L., Papaioannou, A., Ioannidis, G., Levine, M. A., and Adachi, J. D. (2017). An overview of osteoporosis and frailty in the elderly. BMC Musculoskelet. Disord. 18,46. doi: 10.1186/s12891-017-1403-x

Lu, H., Liu, F., Zhu, Q., Zhang, M., Li, T., Chen, J., et al. (2017). Aflatoxin B1 can be complexed with oxidised tea polyphenols and the absorption of the complexed aflatoxin B1 is inhibited in rats. J. Sci. Food Agric. 97, 1910-1915. doi: 10.1002/jsfa.7994

Lv, H. P., Zhang, Y. J., Lin, Z., and Liang, Y. R. (2013). Processing and chemical constituents of Pu-erh tea: a review. Food Res. Int. 53, 608-618. doi: 10.1016/j. foodres.2013.02.043

Macedo, R. M., Brentegani, L. G., and Lacerda, S. A. (2015). Effects of coffee intake and intraperitoneal caffeine on bone repair process-a histologic and histometric study. Braz. Dent. J. 26, 175-180. doi: 10.1590/0103-6440201 300219

Michael Lewiecki, E. (2017). Osteoporosis: treat-to-target. Curr. Osteoporos. Rep. doi: 10.1007/s11914-017-0350-7 [Epub ahead of print].

Nakashima, Y., and Haneji, T. (2013). Stimulation of osteoclast formation by RANKL requires interferon regulatory factor- 4 and is inhibited by simvastatin in a mouse model of bone loss. PLoS ONE 8:e72033. doi: 10.1371/journal.pone. 0072033

Nash, L. A., and Ward, W. E. (2017). Tea and bone health: findings from human studies, potential mechanisms, and identification of knowledge gaps. Crit. Rev. Food Sci. Nutr. 57, 1603-1617. doi: 10.1080/10408398.2014.10 01019

Ng, T. P., Aung, K. C., Feng, L., Nyunt, M. S., and Yap, K. B. (2014). Tea consumption and physical function in older adults: a cross-sectional study. J. Nutr. Health Aging 18, 161-166. doi: 10.1007/s12603-013-0354-7

Oka, Y., Iwai, S., Amano, H., Irie, Y., Yatomi, K., Ryu, K., et al. (2012). Tea polyphenols inhibit rat osteoclast formation and differentiation. J. Pharmacol. Sci. 118, 55-64. doi: 10.1254/jphs.11082FP 
Pan, H., Kopeckova, P., Liu, J., Wang, D., Miller, S. C., and Kopecek, J. (2007). Stability in plasmas of various species of HPMA copolymerPGE1 conjugates. Pharm. Res. 24, 2270-2280. doi: 10.1007/s11095-0079449-3

Park, S. J., Joo, S. E., Min, H., Park, J. K., Kim, Y., Kim, S. S., et al. (2012). Dietary patterns and osteoporosis risk in postmenopausal Korean women. Osong Public Health Res. Perspect. 3, 199-205. doi: 10.1016/j.phrp.2012.10.005

Roux, C., and Briot, K. (2017). Imminent fracture risk. Osteoporos. Int. doi: 10. 1007/s00198-017-3976-5 [Epub ahead of print].

Schwarz, P., Jorgensen, N. R., and Abrahamsen, B. (2014). Status of drug development for the prevention and treatment of osteoporosis. Expert Opin. Drug Discov. 9, 245-253. doi: 10.1517/17460441.2014.884067

Shan, Y., Zhang, M., Wang, T., Huang, Q., Yin, D., Xiang, Z., et al. (2016). Oxidative tea polyphenols greatly inhibit the absorption of atenolol. Front. Pharmacol. 7:192. doi: 10.3389/fphar.2016.00192

Shen, C. L., Chyu, M. C., and Wang, J. S. (2013). Tea and bone health: steps forward in translational nutrition. Am. J. Clin. Nutr. 98, 1694S-1699S. doi: 10.3945/ajcn.113.058255

Shen, C. L., Yeh, J. K., Cao, J. J., Chyu, M. C., and Wang, J. S. (2011). Green tea and bone health: evidence from laboratory studies. Pharmacol. Res. 64, 155-161. doi: 10.1016/j.phrs.2011.03.012

Shen, C. L., Yeh, J. K., Cao, J. J., and Wang, J. S. (2009). Green tea and bone metabolism. Nutr. Res. 29, 437-456. doi: 10.1016/j.nutres.2009.06.008

Tabatabaei-Malazy, O., Salari, P., Khashayar, P., and Larijani, B. (2017). New horizons in treatment of osteoporosis. Daru 25,2 doi: 10.1186/s40199-0170167-z

Vester, H., Holzer, N., Neumaier, M., Lilianna, S., Nussler, A. K., and Seeliger, C. (2014). Green tea extract (GTE) improves differentiation in human osteoblasts during oxidative stress. J. Inflamm. 11:15. doi: 10.1186/1476-9255-11-15

Visagie, A., Kasonga, A., Deepak, V., Moosa, S., Marais, S., Kruger, M. C., et al. (2015). Commercial honeybush (Cyclopia spp.) tea extract inhibits osteoclast formation and bone resorption in RAW264.7 murine macrophages-an in vitro study. Int. J. Environ. Res. Public Health 12, 13779-13793. doi: 10.3390/ ijerph121113779

Wang, S., Huang, Y., Xu, H., Zhu, Q., Lu, H., Zhang, M., et al. (2016). Oxidized tea polyphenols prevent lipid accumulation in liver and visceral white adipose tissue in rats. Eur. J. Nutr. doi: 10.1007/s00394-016-1241-x [Epub ahead of print].

Xie, J., Yu, H., Song, S., Fang, C., Wang, X., Bai, Z., et al. (2017). Pu-erh tea water extract mediates cell cycle arrest and apoptosis in MDA-MB-231 human breast cancer cells. Front. Pharmacol. 8:190. doi: 10.3389/fphar.2017.00190
Xu, H., Wang, Y., Chen, Y., Zhang, P., Zhao, Y., Huang, Y., et al. (2016). Subcellular localization of galloylated catechins in tea plants [Camellia sinensis (L.) O. Kuntze] assessed via immunohistochemistry. Front. Plant Sci. 7:728. doi: 10.3389/fpls.2016.00728

Yu, Y., Hayashi, S., Cai, X., Fang, C., Shi, W., Tsutsui, H., et al. (2014). Pu-erh tea extract induces the degradation of FET family proteins involved in the pathogenesis of amyotrophic lateral sclerosis. Biomed. Res. Int. 2014:254680. doi: $10.1155 / 2014 / 254680$

Zeng, L., Yan, J., Luo, L., and Zhang, D. (2015). Effects of Pu-erh tea aqueous extract (PTAE) on blood lipid metabolism enzymes. Food Funct. 6, 2008-2016. doi: 10.1039/c5fo00362h

Zeng, X. Z., He, L. G., Wang, S., Wang, K., Zhang, Y. Y., Tao, L., et al. (2016). Aconine inhibits RANKL-induced osteoclast differentiation in RAW264.7 cells by suppressing NF-кB and NFATc1 activation and DC-STAMP expression. Acta Pharmacol. Sin. 37, 255-263. doi: 10.1038/aps.2015.85

Zhang, X., Wang, J., Hu, J. M., Huang, Y. W., Wu, X. Y., Zi, C. T., et al. (2016). Synthesis and biological testing of novel glucosylated epigallocatechin gallate (EGCG) derivatives. Molecules 21:E620. doi: 10.3390/molecules21 050620

Zhao, H., Zhang, M., Zhao, L., Ge, Y. K., Sheng, J., and Shi, W. (2011). Changes of constituents and activity to apoptosis and cell cycle during fermentation of tea. Int. J. Mol. Sci. 12, 1862-1875. doi: 10.3390/ijms120 31862

Zhao, L., Jia, S., Tang, W., Sheng, J., and Luo, Y. (2011). Pu-erh tea inhibits tumor cell growth by down-regulating mutant p53. Int. J. Mol. Sci. 12, 7581-7593. doi: 10.3390/ijms12117581

Zou, X. J., Ding, Y. H., and Liang, B. (2012). The mechanisms of weight-cutting effect and bioactive components in Pu-erh tea. Dongwuxue Yanjiu 33, 421-426. doi: 10.3724/SP.J.1141.2012.04421

Conflict of Interest Statement: The authors declare that the research was conducted in the absence of any commercial or financial relationships that could be construed as a potential conflict of interest.

Copyright (c) 2017 Liu, Ding, Yin, Cuan, Xie, Xu, Wang and Sheng. This is an open-access article distributed under the terms of the Creative Commons Attribution License (CC BY). The use, distribution or reproduction in other forums is permitted, provided the original author(s) or licensor are credited and that the original publication in this journal is cited, in accordance with accepted academic practice. No use, distribution or reproduction is permitted which does not comply with these terms. 